\title{
Tolerancia al quebrantamiento de la norma en el area metropolitana de Medellín, Colombia
}

\author{
\begin{tabular}{c}
\hline \hline Luis Fernando Duque \\
Jorge Arbey Toro \\
Nilton Montoya \\
Facultad Nacional de Salud Pública \\
Universidad de Antioquia \\
\hline \hline
\end{tabular}
}

Resumen: El presente artículo analiza la tolerancia al quebrantamiento de la norma en Medellín y municipios del Área Metropolitana a partir de los datos obtenidos del estudio sobre la magnitud, distribución y factores asociados a la violencia en el Área Metropolitana que se realizó en 2004 a los diez municipios que la integran, por el cual se estimó la magnitud que se tiene en la sociedad de este importante factor de riesgo para la violencia. De los resultados se destaca que los indicadores estandarizados de anomia y de legitimación del quebrantamiento de la norma son asombrosamente altos en todos los municipios del Área Metropolitana de Medellín, tanto en hombres como en mujeres, aunque con mayor prevalencia entre los hombres, y entre los menores de edad y los jóvenes. Se presentan discusiones sociológicas que aportan elementos para explicar la presencia y las características de este fenómeno y los factores que motivan a un individuo a ocasionar un incidente que contribuye a dicho fenómeno. Entre los elementos explicativos se mencionan tanto los valores inadecuados o falta de ciertos valores culturales, fruto de condicionantes de índole sociocultural y económica en el Área Metropolitana, como la racionalidad individual.

Palabras clave: anomia; violencia; tolerancia; Medellín; Colombia

\begin{abstract}
This article analyzes the tolerance to the breaches of the norm in Medellin and municipalities in Metropolitan Area. For this purpose, we use data from the study about magnitude, distribution and factors associated with violence in Metropolitan Area of Medellin that was conducted in 2004 to ten of its municipalities. In this study was estimated the magnitude of this important risk factor for violence on society. Results emphasize that the standardized indicators of anomie and legitimacy to the breach of the rule are surprisingly high in all municipalities in the Metropolitan Area of Medellin, for both men and women, although most prevalent among men, children and youth. Sociological arguments are presented to provide elements that explain the presence and characteristics of this phenomenon and the factors that motivate a person to cause an incident that contributes to this phenomenon. Among the explanatory values are mentioned both inadequate and lack of certain cultural values, the result of sociocultural constraints and economic situation as individual rationality.
\end{abstract}

Keywords: anomia; violence; tolerance; Medellín; Colombia 


\section{Introducción}

El informe de un grupo de estudio auspiciado por COLCIENCIAS (GOMEZ, 1999) que acometió la tarea de buscar el factor fundamental que nos caracteriza como nación y que nos distingue de las demás naciones vecinas, concluyó que el rasgo característico de los colombianos es el contraste entre una gran racionalidad individual y una deficiente, casi inexistente, racionalidad colectiva, (LOPEZ e BETANCUR, 1999). Definición que es mas explicitada en dicho libro por el Prof. Boris Salazar quien define esta característica sustantiva de los colombianos como:

"El conjunto de reglas de interacción social que los colombianos seguimos, y hemos venido siguiendo, con mayor o menor entusiasmo, por un largo tiempo. ¿Qué las hace tan especiales? Estar basadas en un desequilibrio tan marcado entre lo privado y lo público, entre lo individual y lo colectivo, que ha logrado crear ese ser extraordinario que es el colombiano de hoy, capaz de enfrentar en forma individual todas las situaciones, de sobresalir en cualquier medio, de sobrevivir en las condiciones más adversas, pero incapaz de crear lazos duraderos con sus compatriotas, de construir empresas comunes, de hacerse creíble para los otros, de creer, también, en los otros (...) Desde esta perspectiva, el colombiano de hoy es un modelo de racionalidad individual: utiliza todas las oportunidades económicas existentes, trata de usar toda la información disponible, actúa en forma sistemática de acuerdo con unos fines de apropiación y supervivencia muy claros. $Y$ esa racionalidad individual se refuerza por la debilidad complementaria de todas las instituciones públicas, por la casi inexistencia de arreglos colectivos creíbles y actuantes. La debilidad de lo público y la fortaleza inusual de lo privado y de lo individual se refuerzan, entonces, mutuamente, hasta llegar al punto en que lo privado, como forma de comportamiento e interacción social deviene, y sustituye, lo público y lo colectivo. Por eso no puede hablarse ni de desorden ni de caos. Por el contrario, nos encontramos ( $y$ ahí está precisamente el desafío) ante un orden de una dureza y de una capacidad de supervivencia extraordinarias, un orden que tiene la virtud terrible de estar firmemente anclado en el comportamiento individual de cada colombiano y de reforzarse todos los día en nuestra interacción" (SALAZAR, 1999). 
Fenómenos como el deseo de ascenso social y de posesión de riqueza tan evidentes en la cultura antioqueña (PRIETO, 1995), y especialmente en la de las zonas urbanas del Área Metropolitana , unidos a una cada vez mayor iniquidad social, caracterizada por la concentración de las oportunidades, han sido caldo de cultivo propicio para que muchos de nuestros niños y jóvenes perciban en forma importante las vías no legales para poder satisfacer sus expectativas e ideales de vida (DUQUE, SIERRA e MONTOYA, 2007a).

\begin{abstract}
"A ello se pueden sumar factores como 'la crisis de representación política; el clientelismo, la corrupción y la impunidad; la instauración de una forma del quehacer político a través de la intimidación y el uso de la fuerza para asegurar lealtades; la falta de presencia territorial y la pérdida de legitimidad del Estado; el debilitamiento del imperio de la ley; la ausencia de una política de tierras, y otros problemas estructurales...' (GARAY, 1999a), un aparato jurídico puramente excepcional y estratégico..., las decisiones judiciales son acogidas cuando éstas son útiles, pero se cuestionan y se deslegitiman cuando afectan los propios intereses. Situación que se debe en gran medida a que los ciudadanos no saben con exactitud qué normas los gobiernan y cuáles son los valores que dichas normas promueven". (GARAY, 2002b; QUINTERO, 2007)
\end{abstract}

En una sociedad con tales características, y muy probablemente por tenerlas, surgió con fuerza el narcotráfico, y muy especialmente en municipios del Área Metropolitana. La cultura mafiosa que reproduce y potencializa en su máxima expresión esa cultura, no fue ni ha sido la causa única de la descomposición social colombiana y de nuestros municipios.

El surgimiento de la cultura mafiosa está también íntimamente ligado al y ha reforzado el desprecio por lo social, comunitario o público; ha tenido como medio de cultivo y ha reforzado la tolerancia por el quebrantamiento de la norma para obtener el mejoramiento individual, y se ha nutrido y ha reforzado también la poca valoración de los derechos de los demás. Estas características se asocian a una aculturación rentística y de la ilegalidad y a la violencia (CAMACHO, LEAL, 2001; GUZMÁN, 2001; MELO, 1995).

La delincuencia organizada, y la vinculación o relación que con ella se tenga, se ha asociado íntimamente con la legitimación de la consecución fácil del dinero, y ambas han sido descritas como asociadas de una manera muy fuerte con la violencia, especialmente la homicida, particularmente en Medellín y en Cali 
(DUQUE, SIERRA, e MONTOYA, 2007a; GARAY, 1999a; SALAZAR, 1992; GUZMÁN, 1993).

Después de llevar a cabo análisis de regresión logística teniendo en cuenta más de una veintena de factores asociados (de protección y de riesgo) relacionados con la persona, la familia, el barrio y la sociedad, hemos documentado que en Medellín y municipios del Área Metropolitana, la anomia y la tolerancia al quebrantamiento de la norma están íntimamente asociadas a diferentes formas de violencia interpersonal: robo, engaño y estafa, amenazas severas (secuestro, extorsión y desplazamiento forzado), amenaza con arma, agresión con arma. La razón de momios o de oportunidades de tales factores (anomia y tolerancia al quebrantamiento de la norma) fue mayor de 2,0 en todos los casos de violencia interpersonal mencionados. Esto es, que quien posee estas características tiene el doble de probabilidades de ser agresor por las formas de violencia mencionadas que quien no las tiene, (DUQUE, SIERRA, MONTOYA, 2007a).

Estas consideraciones nos han animado a estimar el grado de tolerancia por el quebrantamiento de la norma que tienen los habitantes de los diez municipios con asiento en el Área Metropolitana, con el fin de estimar la magnitud que se tiene en la sociedad de este importante factor de riesgo para la violencia: la tolerancia al quebrantamiento de la norma.

\section{Los datos de la investigación}

En 2003 y 2004 se llevó un estudio de prevalencia sobre la magnitud, distribución y factores asociados a la violencia en los diez municipios del Área Metropolitana de Medellín por el Programa PREVIVA de la Universidad de Antioquia. ${ }^{1}$ La información en la que se basa este artículo proviene de dicho estudio, que se llevó a cabo por medio de entrevista personal a una muestra representativa de la población urbana, no institucional de 12 a 60 años del casco urbano de los diez municipios del Área Metropolitana. El tamaño muestral fue de 5.781 encuestados, y las características de la muestra y de la encuesta se han presentado en otras publicaciones (DUQUE, 2005b; PROGRAMA PREVIVA, 2006).

Al llevar a cabo el análisis de clúster jerárquico de las diferentes variables que reflejan la tolerancia frente al quebrantamiento de la norma, las preguntas se

\footnotetext{
${ }^{1}$ Estudio auspiciado por la Universidad de Antioquia y el Área Metropolitana del Valle de Aburrá. El Valle de Aburrá es una de las nueve regiones del Departamento de Antioquia. Está conformada por la ciudad capital, Medellín, y nueve municipios más: Bello, Envigado, Itagüí y Sabaneta (urbanos), La Estrella, Girardota, Barbosa, Caldas, Copacabana (semi urbanos). La población del Valle de Aburrá es de 3'312.165 habitantes (2005), que equivalen al 59,4\% de la población de Antioquia y al 8,0\% de la de Colombia
} 
agruparon en cuatro variables de resumen que hemos denominado de la siguiente manera:

\section{1) Aprobación de conseguir dinero por las vías no legales}

- Transporte de droga al extranjero si se necesita dinero, con un peso de 0,0800

- Volverse rico participando en negocios ilegales, con un peso de 0,0996

- Llevarse cosas (papelería, útiles de oficina, elementos de aseo, etc.) de la empresa donde trabaja, con un peso de 0,0817

- Quedarse con una billetera que alguien dejó por ahí, con un peso de 0,0671

- Mandar un matón para arreglar cuentas con alguien que no le quiere pagar una cantidad grande de dinero, con un peso de 0,0867

\section{2) Aprobación de corrupción al funcionario público}

- Dar dinero a un funcionario o autoridad pública para evitarse una multa o agilizar un trámite, con un peso de 0.0488

- Pagar a alguien para conseguir la libreta militar del hijo, con un peso de 0,0097

3) Aprobación de la violencia extrema en defensa de la familia o en beneficio político y económico

- Secuestrar a alguien con un fin político, con un peso de 0,0822

- Secuestrar a alguien con un fin económico, con un peso de 0,0821

- Matar a un hombre que ha violado a su hija, con un peso de 0,0241

\section{4) Aprobación de uso del asesinato para defensa de la comunidad}

- Eliminar a alguien que la mayoría de la gente cree que pone en riesgo la comunidad, con un peso de 0,0482

- Hacer "limpieza social", es decir, a matar pandilleros, gamines, prostitutas, homosexuales, indigentes, etc., con un peso de 0,0537

Para realizar comparaciones entre diferentes poblaciones se construyó un indicador estandarizado de tolerancia frente al quebrantamiento de la norma, con base en el peso que tiene cada una de las preguntas en la variable de resumen y en la puntuación que se dio a cada una de las categorías de respuesta que se presenta en la escala de Likert: muy en desacuerdo $=0$, algo en desacuerdo $=1$, algo de acuerdo $=2$ y muy de acuerdo $=3$. El valor de $p$ se calculó para diferencia de múltiples porcentajes usando el test Chi Cuadrado cuando se comparan tres o más proporciones y el Test $Z$ cuando se trata de encontrar diferencias entre dos proporciones, para la diferencia del indicador estandarizado de las variables resumen tratadas en este capítulo se utilizo la prueba $T$ de Student para grupos independientes o U de Mann Witney, según el caso. 
Las características técnicas de la encuesta, cuya base de datos se analiza en este aspecto en el presente documento se hallan en otras publicaciones. (DUQUE, 2005b; PROGRAMA PREVIVA, 2006)

\section{Los resultados}

En primer lugar se presentan los resultados de los indicadores estandarizados de las cuatro variables de resumen o clusters que expresan la actitud de tolerancia frente al quebrantamiento de la norma. Los valores estandarizados pueden estar entre 0 , cuando a todas las preguntas de cada variable de resumen se hubiera respondido que se está totalmente en desacuerdo, y 100, cuando a todas las preguntas de una variable de resumen se hubiera respondido estar totalmente de acuerdo.

En segundo lugar se presentan los valores del porcentaje de personas que aprobó en algún grado el quebrantamiento de alguna norma con el fin de ilustrar al lector sobre la magnitud de este fenómeno en nuestra sociedad.

\section{1) Indicadores estandarizados}

El Gráfico 1 presenta los valores de los indicadores estandarizados por sexo. La mayor aprobación se refiere a la aprobación de la corrupción a funcionarios públicos (soborno), seguida de la aprobación del uso del asesinato en defensa de la comunidad. En general, los indicadores de tolerancia frente al quebrantamiento de la norma son preocupantemente altos.

Los hombres aprueban significativamente más que las mujeres todas las formas de quebrantamiento de la norma, con diferencias entre grupos de edad que son significativas con respecto de aprobación de conseguir dinero por vías no legales $(p=0,0000)$, aprobación de uso de la violencia extrema (homicidio) en defensa de la comunidad $(p=0,0000)$ y aprobación de la corrupción a funcionarios públicos $(p=0,0000)$. En las dos primeras variables enunciadas los mayores indicadores se reportan entre los menores de edad (Gráficos 1 y 2). 


\section{Gráfico 1}

Índice estandarizado de las cuatro variables de resumen sobre la tolerancia frente al quebrantamiento de la norma de los habitantes del Valle de Aburrá entre 12 y 60 años segun el sexo, 2004.

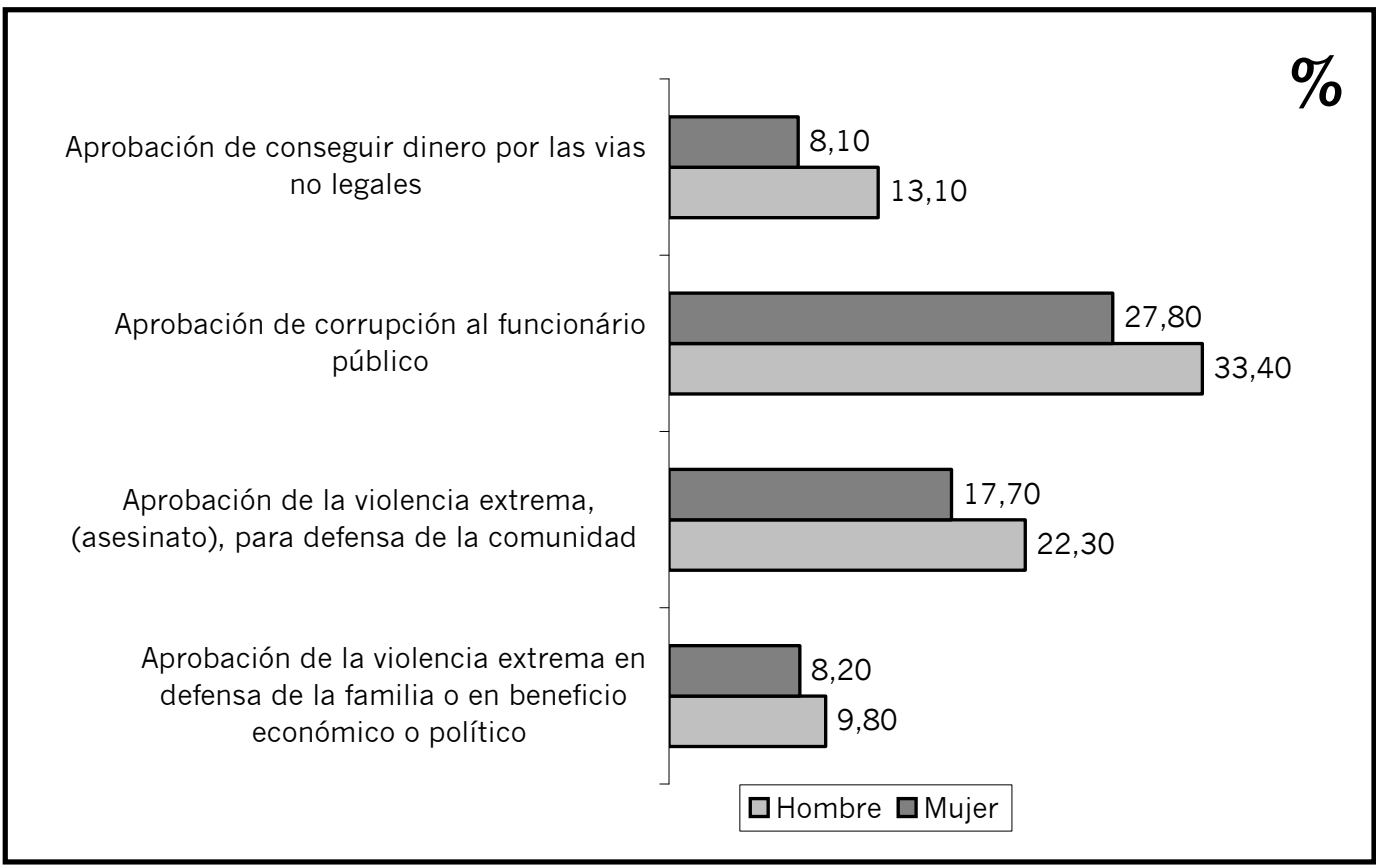




\section{Gráfico 2}

Índice estandarizado de las cuatro variables de resumen sobre la tolerancia frente al quebrantamiento de la norma de los habitantes del Valle de Aburrá entre 12 y 60 años según el grupo de edad, 2004.

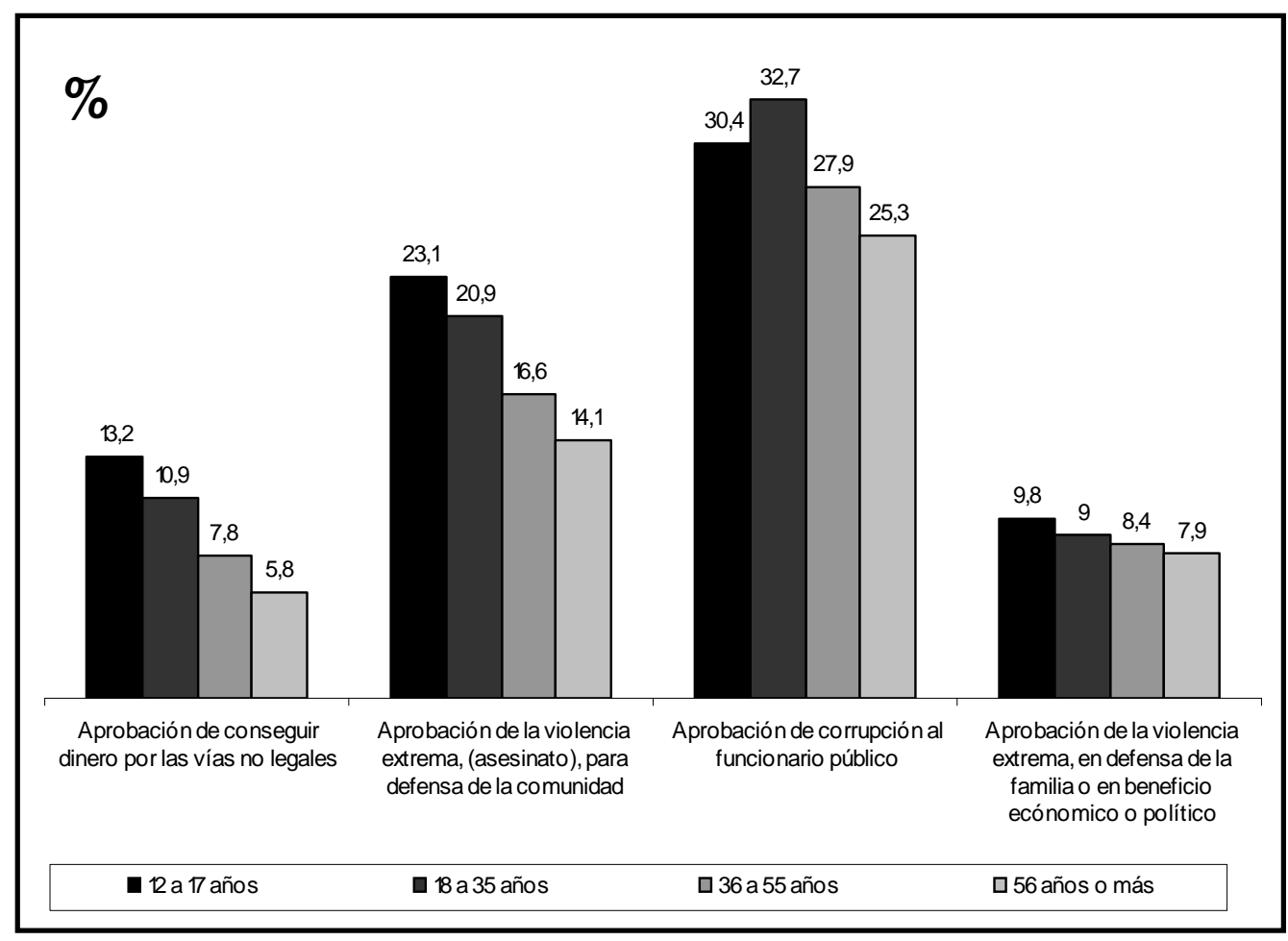

Al repasar los datos por estrato económico y social salta a la vista que en todos los estratos la mayor tolerancia se refiere a la tolerancia a que se corrompa al funcionario público, que es prácticamente igual en todos los estratos ( $p=$ 0,5046). Para los demás indicadores hay una relación inversa con el estrato económico y social. (Cuadro 1). 


\section{Cuadro 1}

Índice estandarizado de las cuatro variables de resumen sobre la tolerancia frente al quebrantamiento de la norma de los habitantes del Valle de Aburrá (12 y 60 años), según el nível económico y social, 2004.

\begin{tabular}{|l|c|c|c|c|}
\cline { 2 - 5 } \multicolumn{1}{c|}{} & Bajo & Medio & Alto & Valor p \\
\hline $\begin{array}{l}\text { Aprobación de conseguir dinero por las vias no } \\
\text { legales }\end{array}$ & 11 & 10 & 6 & 0,0000 \\
\hline $\begin{array}{l}\text { Aprobación de la violencia extrema, } \\
\text { (asesinato), para defesa de la comunidad }\end{array}$ & 21 & 19 & 17 & 0,0154 \\
\hline $\begin{array}{l}\text { Aprobación de la violencia extrema, em defesa } \\
\text { de la familia o en beneficio económico o } \\
\text { político }\end{array}$ & 10 & 9 & 7 & 0,0193 \\
\hline $\begin{array}{l}\text { Aprobación de corrupción al funcionário } \\
\text { público }\end{array}$ & 30 & 30 & 32 & 0,5046 \\
\hline
\end{tabular}

2) Porcentaje de personas que aceptan el quebrantamiento de la norma

En esta sección se presenta el porcentaje de personas que toleran las manifestaciones de quebrantamiento de la norma. El dato correspondiente a cada pregunta es el porcentaje de personas que manifestó aceptación del quebrantamiento de la norma en algún grado y el valor correspondiente a cada variable de resumen o factor es la proporción de personas que manifestó tolerancia en al menos una de las preguntas que componen la variable de resumen. Los datos de las variables de resumen y su denominación se presentan en caracteres resaltados.

Entre los habitantes del Área Metropolitana de Medellín se observó que hay proporciones asustadoramente altas de tolerancia frente a la desviación de la norma:

i. Mas del $40 \%$ tolera que se consiga dinero por algún medio ilegal o antiético o que se soborne a un funcionario público (si no se tiene en cuenta el soborno para conseguir la libreta militar de un hijo, lo que es tolerado por las tres cuartas partes de los habitantes), o que se elimine (léase se asesine) a alguien que la gente cree que pone en peligro a la comunidad.

ii. Uno de cada cuatro o cinco habitantes del Área Metropolitana tolera que se transporte droga al extranjero si se necesita dinero, se quede con una billetera de otra persona, o se haga "limpieza social" (léase se asesine a pandilleros, gamines, prostitutas, indigentes, etc.).

iii. El $6 \%$ de la población tiene algún grado de aprobación del secuestro. 
Gráfico 3

Porcentaje de personas (12 - 60 años) que tolera comportamientos que quebrantan la norma. Municipios del Valle de Aburrá. 2004.

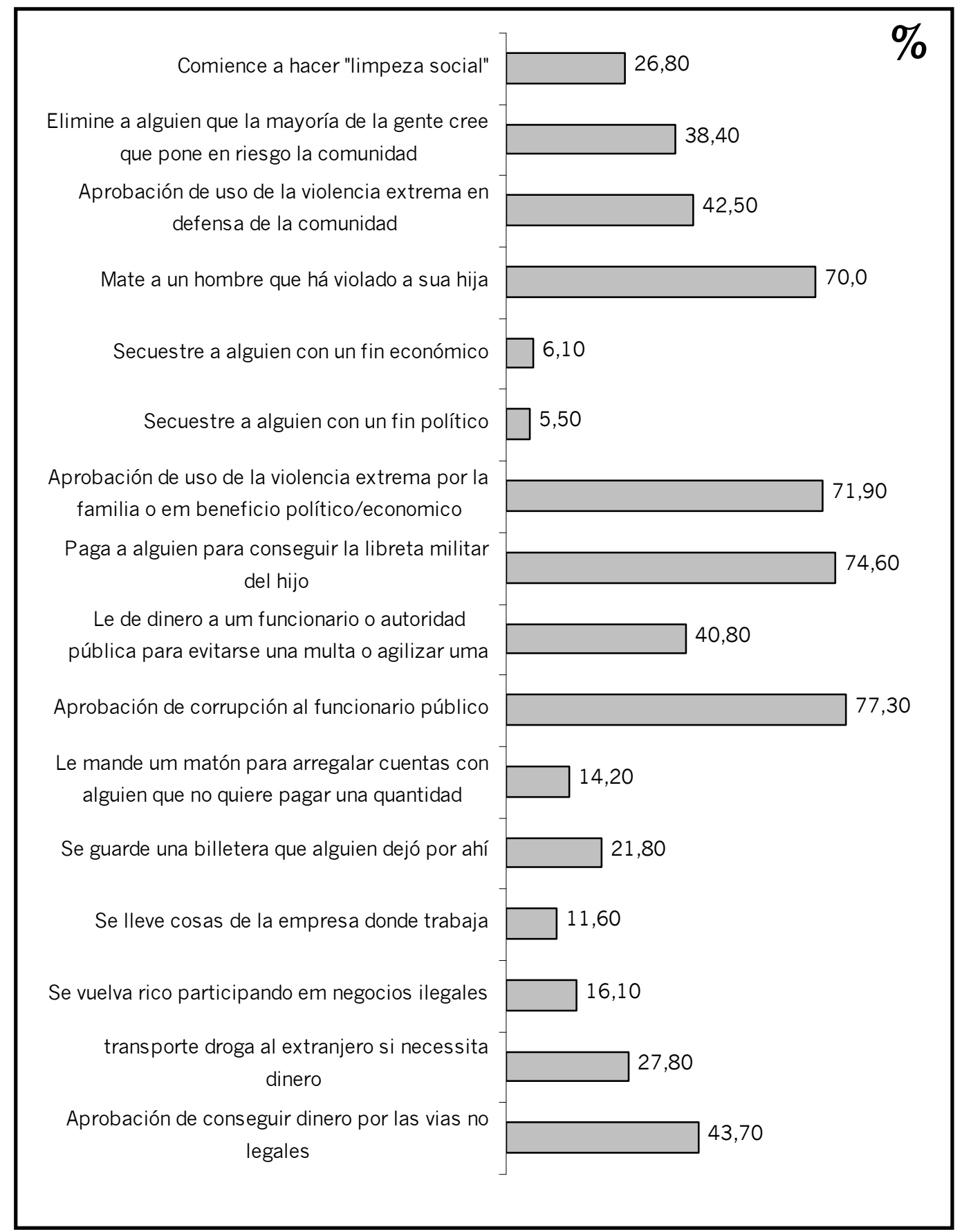


Se observó que el hombre tiene proporciones significativamente más altas de tolerancia frente al quebrantamiento de la norma que la mujer ( $p$ 0,000 a 0,0001).

\section{Gráfico 4}

Porcentaje de personas (12 - 60 años) que tolera comportamientos que quebrantan la norma en el Valle de Aburrá, según el sexo, 2004.

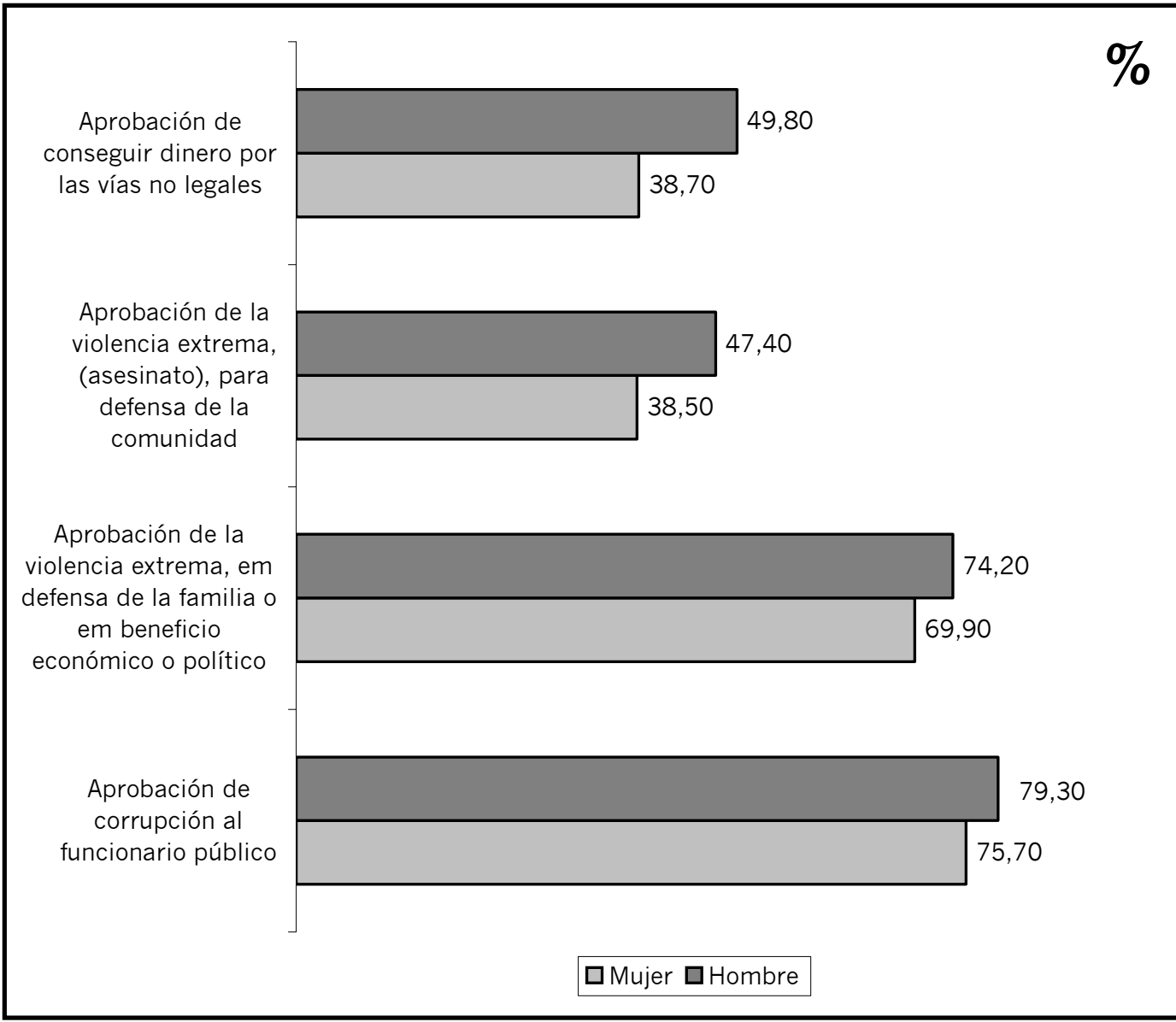

De gran preocupación es que entre los menores de edad y los jóvenes se presenta la mayor proporción de tolerancia de quebrantamiento de las normas, excepto en lo referente a sobornar a un funcionario público. 
Gráfico 5

Porcentaje de personas ( 12 - 60 años) que tolera comportamientos que quebrantan la norma en el Valle de Aburrá, según el grupo etario, 2004.

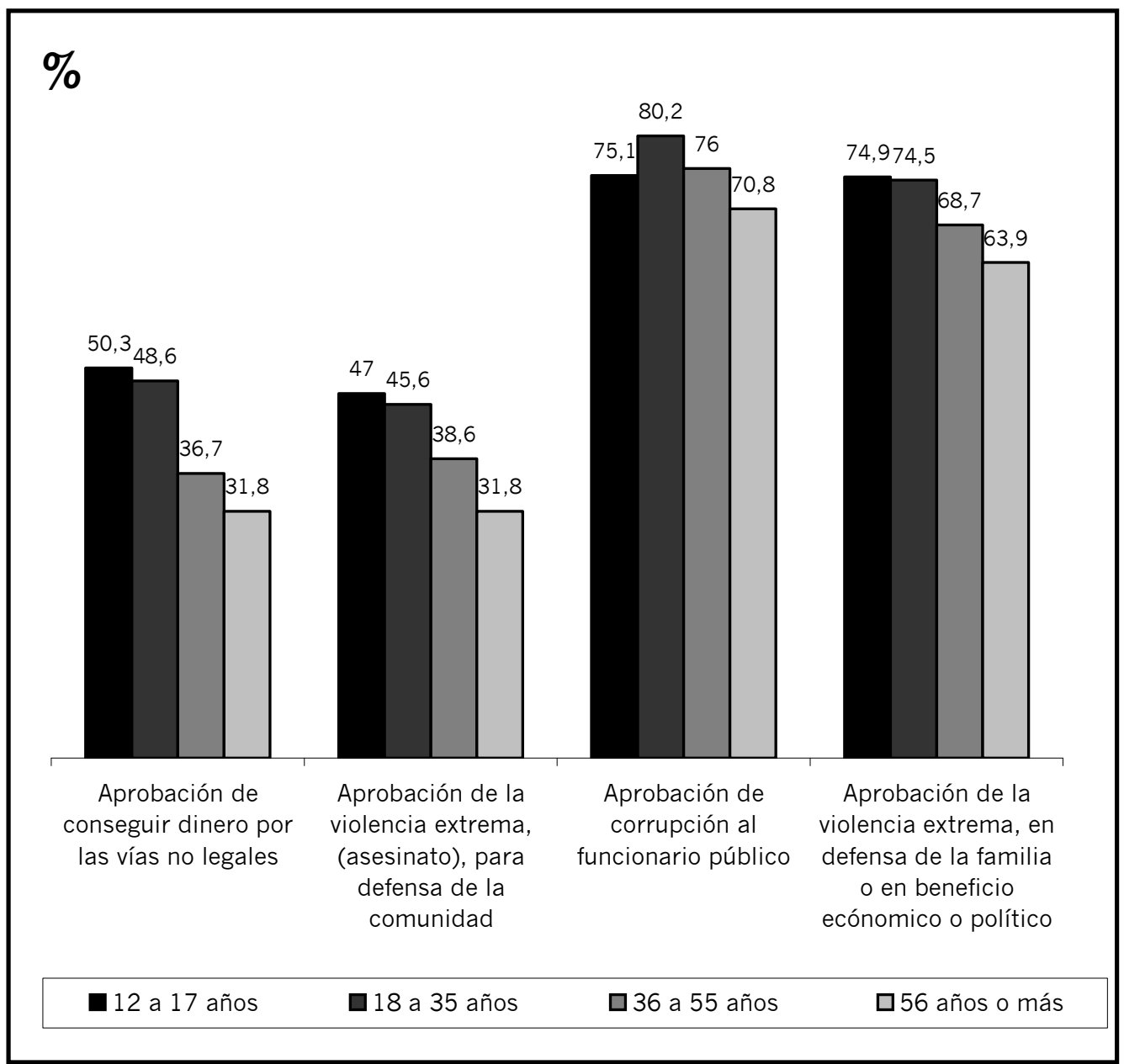

Entre los estratos económicos y sociales solamente hay diferencia significante en cuanto a la tolerancia de conseguir dinero por medios no legales $(p=0,006)$. Las demás diferencias no son de significación estadística. 


\section{Gráfico 6}

Porcentaje de personas (12 - 60 años) que tolera comportamientos que quebrantan la norma en el Valle de Aburrá, según el nivel económico y social, 2004.

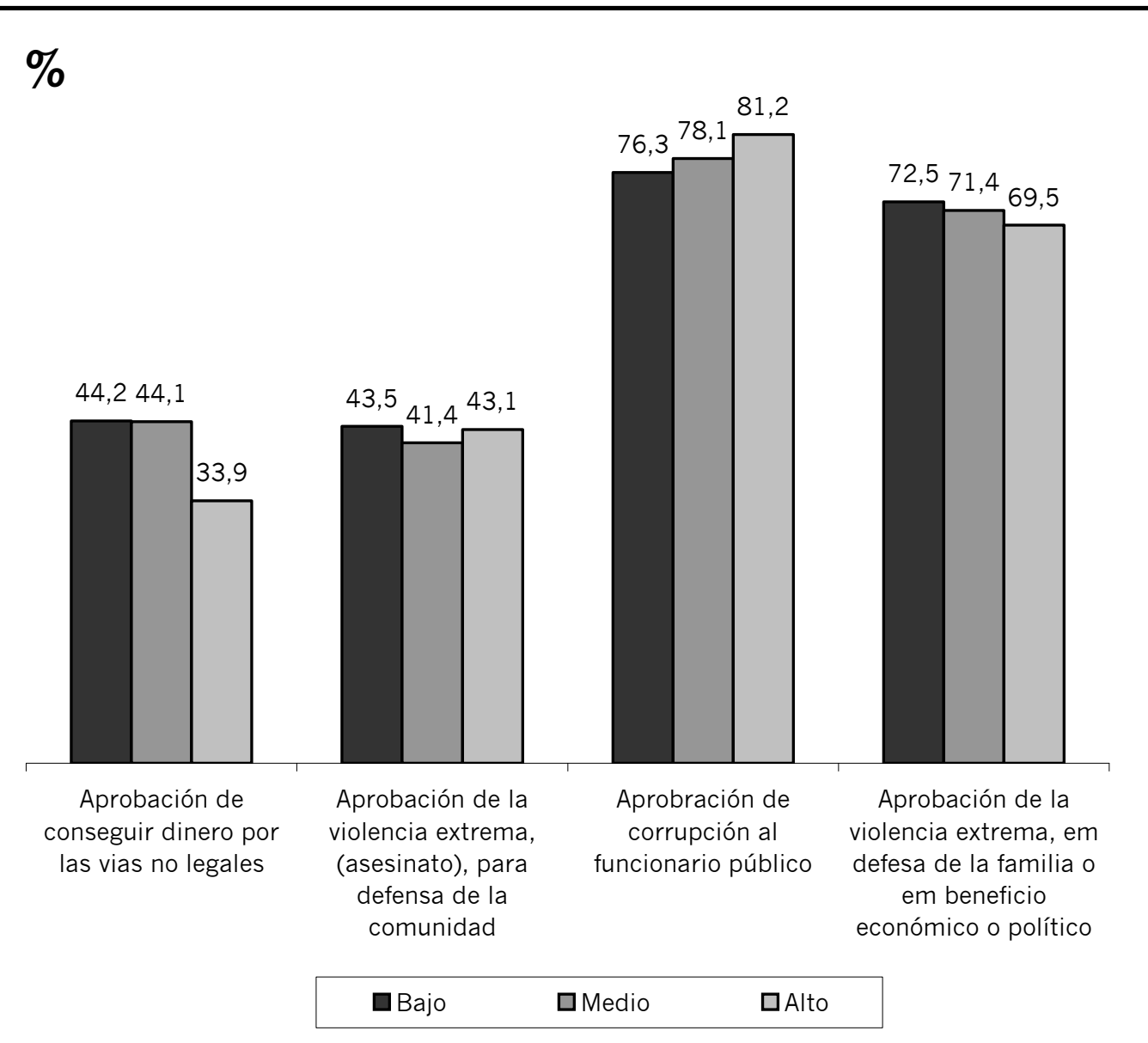

\section{Discusión}

Siguiendo las sugerencias de Durkheim (DURKHEIM, E, 1999), es conveniente hacer énfasis en la necesidad de distinguir entre elementos que ayudan a explicar la presencia y las características sociales de un fenómeno y los factores que motivan a un individuo a ocasionar un incidente que contribuye a dicho fenómeno. En el caso de la tolerancia al quebrantamiento de la norma, es conveniente diferenciar aquellos elementos que ayudan a explicar por qué los habitantes de los diez municipios del Área Metropolitana de Medellín presentan 
unos indicadores estandarizados de legitimación del quebrantamiento de la norma asombrosamente altos, tanto entre los hombres como entre las mujeres, con mayor probabilidad entre hombres que entre mujeres, y son también gravemente altas las proporciones de tolerancia al quebrantamiento de la norma entre los menores de edad y los jóvenes.

Dentro de los elementos explicativos cabe mencionar, aunque no como resultado de un proceso terminado aún, algunos denominados "valores inadecuados" o la falta de ciertos valores culturales, fruto de condicionantes de índole sociocultural y económica en el Área Metropolitana y también la racionalidad individual:

a) Valores inadecuados o falta de ciertos valores culturales.

Aunque la literatura reciente sobre la conducta violenta y delictiva (LARA, 2005) es muy variada, es factible tener una visión general a nivel agregado del fenómeno desde la teoría sociológica. Al respecto, siguiendo el enfoques sociológico de la teoría de la asociación diferencial, que describe el concepto de la desviación social como "la conducta delictiva que se aprende en asociación con aquellos que definen esa conducta favorablemente y aisladamente de aquellos que la definen desfavorablemente" (SUTHERLAND, 1999); y referida, a ciertos estados de vacío o carencia de normas en una sociedad, que provoca, entre otras secuelas, una conducta desviada de algunos de sus miembros, la que, según Merton, "es una ruptura entre fines sociales y los medios para alcanzarlos." (MERTON, 1938) Conducta delictiva que se presenta como la discrepancia entre las expectativas, pues "la conducta anómala puede considerarse desde el punto de vista sociológico como un síntoma de disociación entre las aspiraciones culturalmente prescritas y los caminos socialmente estructurales para llegar a dichas aspiraciones..."(MERTON, 1970).

Podemos colegir que los resultados del estudio son importantes, puesto que permiten validar que la presencia de la violencia en la vida cotidiana de los habitantes de los diez municipios del Área Metropolitana está asociada a la existencia de valores inadecuados o a la falta de ciertos valores culturales (FRANCO V, 2001), es decir, un déficit de valores y normas favorables a la convivencia pero en pro de la desobediencia a las normas sociales básicas, las que son el conjunto de obligaciones, valores y expectativas interpersonales que caracterizan ciertas relaciones de intercambio en la sociedad (PUTNAM, 1993; FRIAS, LÓPEZ, DÍAZ, 2003; CAMACHO, GUZMÁN, 2003).

Esta pérdida de valores, especialmente en los jóvenes, ha sido considerada producto del narcotráfico y representada en las diferentes actividades desarrolladas en la cultura mafiosa y en las transformaciones que representan una 
ruptura con los patrones tradicionales de reconocimiento de los viejos valores de la sociedad antioqueña, como "el trabajo arduo y el ser agente de promoción económica y movilidad social." (RESTREPO, 1996; BOTERO, 1996a; FRANCO, 2006).

Nosotros nos preguntamos si mas bien el narcotráfico floreció en Medellín debido a que en la sociedad se presentaban una serie de características culturales como la tolerancia por el quebrantamiento de la norma, la iniquidad social, el deseo de superación y de mejor ingreso económico, la capacidad de gestión administrativa de los grupos delincuenciales y, como lo resalta Gaitán Daza, (GAITAN, 1995) la ineficiencia en el sistema de justicia. Estas circunstancias fueron propicias para que en Medellín se desarrollara el mayor cartel de la droga que ha existido en Colombia.

El auge del fenómeno del narcotráfico en la ciudad puede haber agudizado que los habitantes del Área Metropolitana y en especial a los jóvenes sean ciudadanos no solidarios, guiados por el interés propio y que legitiman el quebrantamiento de las normas. Según, la Federación Nacional de Comerciantes, FENALCO,

“..., la práctica del enriquecimiento fácil y la falta de compromiso y solidaridad ante la comunidad que critica el origen de los dineros de la nueva clase y el comportamiento de ésta, aunque busca afanosamente su relación de negocios con ella, hace perder una serie de valores tradicionales y de lograr modificaciones sustanciales en la estructura de la comunidad." (EL COLOMBIANO, 1980)

El narcotráfico, según lo evidencian estudios de comienzos de los años noventa, operó como un factor de movilidad económica ascendente en un momento de crisis económica (RESTREPO, 1992). Gestó muchos de los procesos que se desarrollaron más adelante, particularmente, en relación con la instauración de ciertas prácticas nutridas de referentes mafiosos que, en efecto, no han desaparecido de la conflictividad actual, y aunque revestidas de nuevas formas hoy, podrían explicar muchas de las conflictividades urbanas. (SALAZAR y JARAMILLO, 1992; JARAMILLO et al, 1998; RANGEL, 2000; MEDINA, 2006; BLAIR, E, GRISALES y MUÑOZ, 2008).

Otro elemento que debe considerarse, es el trastrocamiento de los patrones de autoridad de la familia matriarcal y su pérdida de centralidad. A comienzos de la década del noventa, con la proliferación de los conflictos por territorio en la ciudad de Medellín, cierta intelectualidad argumentó y difundió con el beneplácito de muchos el discurso del quebrantamiento de patrones de 
autoridad, con el fin de explicar la agudización de las violencias urbanas. Planteaban, que esa fractura era en efecto una ruptura en el ethos cultural del antioqueño, basado hasta entonces en el trabajo, el ahorro, la familia y la religión. En concordancia con esa lectura, ese rompimiento fue atribuido a la secularización y al debilitamiento de la influencia católica, desencadenados por la migración del campo a la ciudad, que trajo consigo, una pérdida de valores. El quiebre de ese ethos cultural antioqueño fue entendido también como el debilitamiento de los mecanismos de control social, que estaban centrados en la religión y la familia. Aquello dejó como resultado "[...] una sumatoria de individualidades que sin cohesión y al vaivén de la historia iba actuando de acuerdo con las urgencias del momento. Una sociedad civil desarticulada, sin proyecto de ciudad alguno." (MEJÍA, 1992).

Hemos documentado que la legitimación de la violencia y la anomia son unos de los factores mas fuertemente asociados a diversas formas de violencia en Medellín y el Área Metropolitana, entre las que se encuentran robo, engaño y estafa, agresión física con y sin arma y amenazas severas, como extorsión, amenaza de secuestro y de desplazamiento forzado (DUQUE, 2009). También hemos hallado que el creer que el desarrollo personal y social debe lograrse por las vías legales, es decir, de acuerdo con las normas está mas asociado a la relación con la madre y el padre y a que ellos sean vigilantes del proceder de sus hijos, que a cualquier otro de los factores estudiados por nosotros. Por el contrario, creer que el desarrollo personal y social debe lograrse por las vías ilegales, es decir, de a contrapelo de las normas está mas asociado a la percepción de iniquidad social y económica (DUQUE, 2009).

b) Racionalidad individual

La tolerancia del quebrantamiento de la norma también tiende a constituirse en un sistema que asegura la subsistencia, pues muchos la ven como la única vía para ser alguien, ejercer un rol y hasta disponer de un lugar en la sociedad, pues la racionalidad individual no implica necesariamente la racionalidad colectiva (QUINTERO, 2007) y da fundamento para que el reconocerse excluido o empobrecido, permita tolerar que se consiga dinero por algún medio ilegal o anti-ético, que se soborne a un funcionario público, que se asesine a alguien que la gente cree que pone en peligro a la comunidad, además de tolerar que se transporte droga al extranjero si se necesita dinero, que se quede con una billetera de otra persona, o se haga "limpieza social".

Lo cierto es que en Medellín y el área metropolitana la tolerancia al quebrantamiento de la norma existe y en alto grado. $Y$ es también cierto que los desfavorecidos socialmente tienden a desviarse más de la norma. Sin embargo 
se ha documentado que en Medellín y el Área Metropolitana tanto agresores o criminales severos como los que no lo son consideran que la sociedad les brinda oportunidades para llegar a ser lo que anhelan en la vida, también perciben que las personas de estrato económico y social alto encuentran en la sociedad más probabilidades de llegar a ser lo que desean ser en la vida que los de estrato bajo, pero que estos, si acceden a educación superior de calidad, encuentran oportunidades similares a las que tienen las personas de estrato alto. Sin embargo los agresores o criminales consideran que en sus circunstancias concretas de vida es necesario acudir a conductas proscritas por las normas para poder colmar sus aspiraciones de vida (DUQUE, MONTOYA, MONTOYA, 2007c).

Lo que claramente indica que entre los agresores o criminales severos la ubicación social deficitaria es percibida como un obstáculo para tener al alcance los medios catalogados como normales para el logro de los fines estándar previstos en la sociedad, pero que una educación superior de calidad es un mecanismo sentido como vía de superación personal y cabe entonces deducir, como se ha dicho, que la tolerancia y aceptación de los fines sin importar los medios (anomia) es lo que induce a la búsqueda de medios alternativos, incluso desviados de la norma.

Lo anterior, supone que la racionalidad individual permite en las personas saber evaluar sus propios intereses, lo que en una sociedad como la nuestra no es poco, pues el principio de racionalidad interpreta las acciones individuales como acciones intencionales de los individuos. "Al no poder adquirir los bienes de consumo..., por el trabajo y el ahorro, se opta por los caminos proscritos" (BRICEÑO . LEÓN y ZUBILLAGA, 2001), entre los cuales se encuentra el quebrantamiento de la norma como una alternativa privilegiada que se generaliza cada vez mas:

"Cuando es obvio que muchos violan la ley sin que implique costo alguno, la lección que se extrae erosiona aún más la predictibilidad de las relaciones sociales; la ilegalidad y el oportunismo generalizados aumentan toda clase de costos de transacción y la texturización de la sociedad por el Estado, en tanto ley, se debilita con cada vuelta de espiral". (O'DONNELL, EN MACK, et al, 2006).

Crudamente, la aceptación del quebrantamiento de las normas, se estaría abriendo terreno en la "legitimidad", en una sociedad desestructurada y con un fuerte individualismo en la que "todo el mundo compite con todo el mundo" (THOUMI, 2008).

La aprobación del uso de la violencia extrema, en defensa de la familia o en beneficio económico o político, se presenta como valor positivo "legítimo" que 
caracteriza a quienes justifican el quebrantamiento de la norma por razones culturales (los demás lo hacen..., es lo acostumbrado..., alguien ejemplar lo ha hecho...)... o de utilidad inmediata (gran provecho económico..., única manera de alcanzar el objetivo..., alguien lo ha hecho con éxito...) (MOCKUS y CORZO, 2003).

En el mismo sentido Duque explica que cuando una persona o un grupo de personas legitiman el uso de la violencia, lo hace porque considera que es un medio mejor que otras formas de conducta para buscar soluciones a sus problemas o necesidades:

"Las personas y las sociedades que tienen un repertorio limitado de alternativas para solucionar problemas o satisfacer necesidades son usualmente agresivas, violentas..., además, que entre nosotros hay una gran asociación entre legitimación del uso de la violencia, anomia y desconfianza en los demás" (DUQUE, 2009d).

Así mismo, la legitimación de la violencia para proteger a la familia o a la sociedad, se manifiesta en actitudes como "creer que una persona tiene derecho a matar para defender su casa o propiedad; que tiene derecho a matar para defender a su familia; que si las autoridades fallan, la gente tiene derecho a hacer justicia por su propia cuenta; que en algunos casos se justifica que la policía torture a los sospechosos para obtener información, o que debería existir la pena de muerte para ciertos crímenes" y se presenta de manera significativamente más alta entre quienes han optado por la agresión y criminalidad que entre las personas del común (controles comunitarios) (DUQUE, MONTOYA, y MONTOYA, 2007c), aunque en estas hemos observado que los valores de tolerancia al quebrantamiento de la norma son sumamente altos.

\section{Conclusión}

Los municipios del Área Metropolitana de Medellín, manifiestan factores negativos con respecto al quebrantamiento de la norma, que además de relacionarse con valores inadecuados o falta de ciertos valores culturales de los colombianos, que según Gómez (GOMEZ, 1985), "en cualquier género de actividades de despliegue [el colombiano] muestra la ...tendencia a mentir y a engañar, porque, se le figura, son condiciones indispensables para el éxito", y esto, se manifiesta con mayor fuerza en esta ciudad, puesto que, con el correr del tiempo la tolerancia al quebrantamiento de la norma, se ha convertido en un problema colectivo, que la ciudad no ha sido capaz de enfrentar con la procura de una mejor la racionalidad pública que sea mas aceptada o legitimada que la racionalidad 
individual. Este es un asunto principalmente de debilidad social, en el sentido que la sociedad se ha vuelto demasiado tolerante con la ilegalidad, a lo que se suma la debilidad estatal para enfrentar este tema (THOUMI, 2002; DUQUE, 2009d).

La proporción de personas que tolera el quebrantamiento de la norma en el Área Metropolitana de Medellín es inquietante. El incumplimiento de la ley no es visto como algo moral o socialmente reprochable. Ese divorcio ha llevado a un auge de la violencia, de la delincuencia y de la corrupción; al desprestigio de las instituciones; al debilitamiento de las tradiciones culturales y a una crisis de la moral individual (MOCKUS, 2003).

Se considera culturalmente aceptable que se consiga dinero por algún medio ilegal, que se asesine a alguien que la gente cree que pone en peligro a la comunidad; algunos comportamientos como sobornar a un funcionario público, transportar droga si se necesita dinero o, quedarse con una billetera de otra persona, son socialmente tolerados.

La sociedad entera, y no meramente el Gobierno o el Estado, tiene en sus manos un severísimo problema, que hace que haya que mirar toda la tarea de prevención y control de la violencia y la delincuencia como una tarea no meramente programas de prevención de la violencia orientados a grupos específicos de la sociedad, que han probado ser efectivos en otras culturas y entre nosotros, sino que para poder prevenir la violencia y la delincuencia entre nosotros es necesario ir más allá: se impone la construcción de ciudadanos comprometidos socialmente. ¡Es necesario emprender la "reingeniería" del antioqueño!

Esta información nos muestra una sociedad enferma, y los factores aquí analizados no están solamente relacionados con la violencia, sino con todo el funcionamiento del aparato social. En suma, la acción que se lleve a cabo para emprender la construcción de ciudadanos, dará frutos no solo de prevención de la violencia, sino de progreso social, cultural y económico. 


\section{Referencias Bibliográficas}

BOTERO F. La planeación del desarrollo urbano de Medellín, 1995-1994. En MELO J O. Historia de Medellín Tomo II. Suramericana de Seguros. 1996, Bogotá: Editorial Norma, 1995.

Medellín: ¿un proyecto realizado o fruto del azar? Una reflexión histórica, en Medellín autores urbanos y proyectos de ciudad. Medellín, Seminario. Corporación Región. Universidad Nacional de Colombia. p. 7.9, Noviembre 10 y 11 de 1994.

BUSS A.H. The psychology of aggression. Nueva York: Wiley; 1961. p. 171 - 173.

BRICEÑO -LEÓN R y ZUBILLAGA V. Dimensiones y construcciones de la violencia en América latina. Acta Científica Venezuela, 52: 172, 2001.

CAMACHO G A y GUZMÁN Á. Colombia, ciudad y violencia. Bogotá: Ediciones Foro Nacional Bogotá, p. 26, 1990.

CONSEJERÍA PRESIDENCIAL PARA MEDELLÍN Y SU ÁREA METROPOLITANA. TERCER SEMINARIO ALTERNATIVAS Y ESTRATEGIAS DE FUTURO PARA MEDELLÍN Y SU ÁREA METROPOLITANA. Medellín, Mimeo, pp, 309-314,1992.

CUBIDES, H; M. LAVERDE Y C. VALDERRAMA (Eds.). Viviendo a toda. Jóvenes, territorios culturales y nuevas sensibilidades. Bogotá: Siglo del Hombre Editores, 1998.

DUQUE, L. F. La violencia en el Valle de Aburrá. Su magnitud y programa para reducirla. Medellín: Universidad de Antioquia y Área Metropolitana del Valle de Aburrá. Fotográficas Mario Salazar; 2005.

DUQUE L. F; MONTOYA N. E e MONTOYA M. Similitudes y diferencias entre miembros de las Autodefensas Unidas de Colombia, agresores severos y controles comunitarios en Medellín. Rev. Fac. Nac. Salud Pública, 2007: 25 (2): 37.47

DUQUE, L. F; KLEVENS J; RAMIREZ C. "Cross-sectional Survey of Perpetrators, Victims and Witnesses of Violence in Bogota Colombia". Bogota: Jornal of Epidemiology community health. 2003; 57:355-60.

DUQUE L. F; KLEVENS, J. “La Violencia en Itagüí, Antioquia (I) : Prevalencia y Distribución.” Biomedica, Bogota, 2000; 20:151-68

DUQUE, LF. e otros. (ed) La violencia en el Valle de Aburrá. Caminos para la superación. Universidad de Antioquia y Área Metropolitana del Valle de Aburrá, Medellín. $2^{a}$ impresión. Medellín: Litoservicios, 2009.

DURKHEIM, E. El Suicidio. México: Ediciones Coyoacán. 5ta. Edición, 1999 [1897]. 
EI COLOMBIANO. Medellín, octubre 30, 1980, p. 10A.

FRANCO, R. V. Practicas hegemónicas de la coalición política dominante. Entre la coerción y el consenso. En FRANCO, R. V. Poder regional y proyecto hegemónico. EI caso de la ciudad metropolitana de Medellín y su entorno regional 1970-2000: IPC, Instituto Popular de Capacitación, Medellín, Colombia: Colombia. 2005. Capitulo 7. Disponible en:

<http://bibliotecavirtual.clacso.org.ar/ar/libros/colombia/ipc/poderregional.pdf> Acesso em: 26 de enero, 2010.

FRANCO V. L. Violencias, conflictos urbanos y guerra civil: El caso de la ciudad de Medellín en la década de los noventa. Conflictos urbanos y alternativas. Compilación. In: Seminario internacional Conflictos Urbanos y Alternativas de transformación. Medellín: IPC. 2004, p. 16

FRIAS A. M; LÓPEZ, E; DÍAZ, M. S. Predictores de la conducta antisocial juvenil: un modelo ecológico. Estud. psicol. (Natal), 2003. 8 (1):15-24.

GAITÁN, D. Colombia un proyecto inconcluso. Colombia un país por construir. Reseña Colombia un Proyecto Inconcluso: valores, instituciones y capital social, de Cuéllar, M. M. Revista de Economía Institucional. Universidad Externado de Colombia, Bogotá. p. 187-193. No 4 Primer Semestre, 2001.

GARAY, L. G. Crisis y construcción de la sociedad: Apuntes para el caso de Colombia. En GÓMEZ BUENDÍA, H, Ed. ¿Para dónde va Colombia? Bogotá: Tercer Mundo Editores - Colciencias, 1999.

GARAY L.J. La transición hacia la construcción de la sociedad: Reflexiones en torno a la crisis colombiana. En: CAMACHO, A, Y LEAL, F. (compiladores). "Armar la paz es desarmar la guerra". CEREC, DNP, FESCOL, IEPRI Universidad Nacional, Misión Social, Presidencia de la República-Alto Comisionado para la Paz. Santa Fe de Bogotá: Giro Editores, 2001.

GARAY, L. J. Repensar a Colombia: Hacia un nuevo contrato social. Bogotá. Agencia Colombiana de Cooperación Internacional - PNUD. 2002. En: QUINTERO, M, M. Justificaciones de jóvenes universitarios y jóvenes desplazados acerca de la concepción de justicia. Centro de estudios avanzados en niñez y juventud. Universidad de Manizales - CINDE. 2006.

GARCIA V, M. Normas de papel: la cultura del incumplimiento de reglas. Bogotá: Siglo del Hombre Editores; Centro de Estudios de Derecho, Justicia y Sociedad. DeJuSticia, 2009.

GÓMEZ B, H, (Ed.). ¿Para dónde va Colombia? Bogotá: Tercer Mundo Editores Conciencias, 1999. 
GOMEZ, A, H. La decadencia del pueblo colombiano. Barcelona: Plaza \& Janes. 1995.

GUZMÁN, A. Diagnóstico de la violencia homicida en Cali. Documentos de Trabajo. CIDSE-Universidad del Valle, Cali, 1993.

GUZMÁN, A. Violencia Urbana: Teorías y Políticas de Seguridad Ciudadana. En: CAMACHO, A. y LEAL, F. (compiladores) "Armar la paz es desarmar la guerra" CEREC, DNP, FESCOL, IEPRI Universidad Nacional, Misión Social, Presidencia de la República-Alto Comisionado para la Paz. Santa Fe de Bogotá. Giro Editores, 2001.

JARAMILLO, A. M; CEBALLOS, M. R. y VILLA M. M. En la Encrucijada. Conflicto y cultura política en el Medellín de los noventa. Medellín, Corporación Región, Secretaria de Gobierno y Programa para la Reinserción - Red de Solidaridad Social. Medellín. Editorial Pregón Ltda., 1998.

LARA, $\mathrm{H}$. Enfoques actuales en la Psicobiología contemporánea de la conducta violenta y delictiva. Revista Neurología, Neurocirugía y Psiquiatría; México, Sociedad Mexicana de Neurología y Psiquiatría, A.C. 2005. 38(1): 28-36.

LÓPEZ M. A; BETANCUR, B. Preámbulo. El despertar nacional. En: Gómez B. H. (Ed.). ¿Para dónde va Colombia? Bogotá, Tercer Mundo Editores - Colciencias. 1999.

MEDINA, F. G. Historia sin fin... Las milicias en Medellín en la década del noventa. Medellín, IPC, .2006.

MEJÍA, M. E. La relación Estado-sociedad civil: el caso de Medellín". En: Consejería Presidencial para Medellín. Op. cit., p. 124

MELO, J. O. Ciudadanía y violencia: Algunas notas sobre la experiencia en Medellín. Boletín Socioeconómico, Universidad del Valle. Cali. CIDSE. No 29. 1995. . Contra la identidad. En: el Malpensante, No 74, 85-98. Nov-Dic, 2006.

. Medellín: historia y representaciones. En: MELO, J. (coord.). Medellín, actores urbanos y proyectos de ciudad. Medellín: Corporación Región/Universidad Nacional de Colombia, Sede Medellín, 1994.

MERTON, R. Estructura social y anomia: revisión y ampliación. American Sociological Review-3, Washington. García-Pablos. Antonio Supra, 1938 p.515 1970. . Teoría y Estructura Sociales. México. Ed. Fondo de Cultura Económica. 
MOCKUS, A y CORZO, J. Dos caras de la convivencia. Cumplir acuerdos y normas y no usar ni sufrir violencia. Análisis político, Bogotá, 2003. n 48, págs. 3-26. enero-abril.

O'DONNELL, G; EN MACK, L. et al., Redes de inclusión: Entendiendo la verdadera fortaleza partidaria, Cuadernos de Información Política, Guatemala, No. 10, FLACSO, 2006. p. 11

ORTIZ, L. P. El (in)cumplimiento de la ley, las instituciones y la cultura en Guatemala. Diálogo, Nueva época. Guatemala. FLACSO Facultad Latinoamericana de Ciencias Sociales, Sede Guatemala. 2008. No. 61.p. 3.

PRIETO, G. Al pie de un volcán. Te escribo. Crónicas latinoamericanas. Bogotá: Norma, 1995.

PROGRAMA PREVIVA. 2006. Medellín. Documento 1. Universidad de Antioquia y Área Metropolitana. Serie de documentos de Información para la Acción para los Comités de Convivencia y Seguridad Ciudadana de los municipios del Área Metropolitana. Presentación de los primeros documentos.

PUTNAM R. Para hacer que la democracia funcione, Caracas: Editorial Galac, 1993.

PUYANA, G. ¿Cómo somos los colombianos? Bogotá: Quebecor World, 2000.

QUINTERO, M.M. Justificaciones y sentimientos morales de jóvenes universitarios y jóvenes desplazados acerca de las acciones justas e injustas. Acta Colombiana de Psicología 2007. 10 (1): 99-110,

RANGEL, A. Parasites and Predators: Guerrillas and the Insurrection Economy of Colombia, Journal of International Affairs, 2000. Vol. 53, Núm. 2, p. 577 a 601

RESTREPO. Y M. Medellín: una ciudad en crisis. Consejería Presidencial para Medellín y su Área Metropolitana. Medellín. Medellín: Alternativas de Futuro, 1992. pp. 309 -314.

SALAZAR, A y JARAMILLO, A. M. Medellín: Las subculturas del narcotráfico. Bogotá: CINEP, 1992

STRAUSS, M. A. Measuring intrafamily conflict and violence: the Conflict Tactic Scales. En: STRAUSS M.A; GELLES R.J. (eds.) Physical violence in American families: risk factors and adaptations to violence in 8145 families. New Brunswick, NJ: Transaction Publications; 1990.

THOUMI, F. Colombia necesita imponer el imperio de la ley. Mimeo, Conferencia: IPC. Medellín, 2008. 
El imperio de la droga: narcotráfico, economía y sociedad en los Andes. Bogotá: Planeta e IEPRI, 2002.

\section{Anexo Metodologico}

Estudio de prevalencia sobre la magnitud, distribución y factores asociados a la violencia en los diez municipios del Área Metropolitana por el Programa PREVIVA de la Universidad de Antioquia Estudio, auspiciado por la Estudio auspiciado por la Universidad de Antioquia y el Área Metropolitana del Valle de Aburra, que se llevó a cabo en 2003/2004 por medio de entrevista personal a una muestra representativa de la población urbana, no institucional de 12 a 60 años del casco urbano de los diez municipios del Área Metropolitana. El tamaño muestral fue de 5.781 encuestados.

La muestra fue seleccionada por etapas: 1) se asignó a cada uno de los municipios con que cuenta el Área Metropolitana una cantidad de encuestas proporcional a su población, 2) utilizando la cartografía del Departamento Nacional de Estadística -DANE, se identificaron y numeraron los sitios donde se cruzan las calles en cada municipio, y se procedió a la selección al azar del número de sitios en el mapa de cada municipio, 3) el equipo encuestador se trasladó a cada uno de dichos sitios de muestreo y elaboró un mapa de los inmuebles, hasta completar tantos cuantos fueran necesarios para llegar a diez o doce inmuebles que fueran viviendas; esta selección se realizó en el sentido de las manecillas del reloj, hasta tener al menos 45 personas, de entre 12 y 60 años, en cada uno de los sitios de muestreo, y 4) entre las personas de 12 a 60 años se seleccionaron al azar 12 personas de cada segmento muestral.

Se llevó a cabo el cálculo de expansión al universo. El factor de expansión se calculó como el inverso del producto de las probabilidades de selección en cada municipio y en cada segmento. Se hicieron pruebas de precisión de la expansión y se compararon los resultados con las proyecciones de población del DANE para los municipios del Área metropolitana de Medellín.

La encuesta se llevó a cabo en el lugar de residencia del encuestado, a quien se le hicieron tres visitas, previa cita concertada, antes de desistir de realizar la entrevista.

Se procuró realizar la encuesta en un lugar tranquilo de la casa ofreciera privacidad. Se empezó con preguntas referentes a generalidades sobre la persona y la familia y, pasado un tiempo en el que se procuraba ganar la confianza del entrevistado, se procedía a las preguntas sobre actos violentos más graves. Al encuestado se le explicaba que su participación era voluntaria y que podía no responder una o varias preguntas o suspender la entrevista en el momento en que 
le pareciera oportuno. Ninguno de los entrevistados se negó a terminar la entrevista.

El formulario fue probado en dos estudios anteriores, uno en Bogotá (Duque LF, Klevens J, Ramírez C., 2000) y otro en la municipalidad de Itagüí. (Duque L F, Klevens, J, 2000) Para el diseño del formulario se tuvieron en cuenta los empleados en otros estudios; (Strauss Ma, 1990; Buss Ah. 1961) una vez elaborado, fue revisado por cuatro expertos para evaluar su validez y comprensión.

Posteriormente fue validado en una muestra por conveniencia por el método de grupos conocidos, que en este caso fueron hombres y mujeres presos por delitos contra las personas y miembros de grupos de meditación trascendental. Se incluyeron los ítems que mostraron adecuada validez discriminante. Para la clasificación del estrato económico y social se empleó la de las Empresas Públicas de Medellín, que figuraba en la cuenta de servicios públicos de la casa de habitación del entrevistado.

Para el análisis se calcularon los intervalos de confianza 95\% para las proporciones de prevalencia y se compararon con una prueba $Z$ para dos proporciones o, según el caso, por medio de la prueba chi cuadrado de diferencia de múltiples proporciones, que indica si entre tres o más proporciones hay una al menos que difiere de las demás.

Al llevar a cabo el análisis de clúster jerárquico de las diferentes variables que reflejan la tolerancia frente al quebrantamiento de la norma, las preguntas se agruparon en cuatro variables de resumen que hemos denominado de la siguiente manera:

\section{Aprobación de conseguir dinero por las vías no legales.}

- Transporte de droga al extranjero si se necesita dinero, con un peso de 0,0800

- Volverse rico participando en negocios ilegales, con un peso de 0,0996

- Llevarse cosas (papelería, útiles de oficina, elementos de aseo, etc.) de la empresa donde trabaja, con un peso de 0,0817

- Quedarse con una billetera que alguien dejó por ahí, con un peso de 0,0671

- Mandar un matón para arreglar cuentas con alguien que no le quiere pagar una cantidad grande de dinero, con un peso de 0,0867 


\section{Aprobación de corrupción al funcionario público.}

- Dar dinero a un funcionario o autoridad pública para evitarse una multa o agilizar un trámite, con un peso de 0.0488

- Pagar a alguien para conseguir la libreta militar del hijo, con un peso de 0,0097

Aprobación de la violencia extrema en defensa de la familia o en beneficio político y económico.

- Secuestrar a alguien con un fin político, con un peso de 0,0822

- Secuestrar a alguien con un fin económico, con un peso de 0,0821

- Matar a un hombre que ha violado a su hija, con un peso de 0,0241

\section{Aprobación de uso del asesinato para defensa de la comunidad.}

- Eliminar a alguien que la mayoría de la gente cree que pone en riesgo la comunidad, con un peso de 0,0482

- Hacer "limpieza social", es decir, a matar pandilleros, gamines, prostitutas, homosexuales, indigentes, etc., con un peso de 0,0537.

Para realizar comparaciones entre diferentes poblaciones se construyó un indicador estandarizado de tolerancia frente al quebrantamiento de la norma, con base en el peso que tiene cada una de las preguntas en la variable de resumen y en la puntuación que se dio a cada una de las categorías de respuesta que se presenta en la escala de Likert: muy en desacuerdo $=0$, algo en desacuerdo $=1$, algo de acuerdo $=2$ y muy de acuerdo $=3$. El valor de $p$ se calculó para diferencia de múltiples porcentajes usando el test Chi Cuadrado cuando se comparan tres o más proporciones y el Test $Z$ cuando se trata de encontrar diferencias entre dos proporciones, para la diferencia del indicador estandarizado de las variables resumen tratadas en este capítulo se utilizo la prueba $T$ de Student para grupos independientes o U de Mann Witney, según el caso.

Luis Fernando Duque - Ifduque@une.net.co

Jorge Arbey Toro - jarbey@hotmail.com

Nilton Montoya·nilton_montoya@yahoo.com

Recebido para publicação em fevereiro de 2009.

Aprovado para publicação em agosto de 2009. 